\title{
A Review of Fine Registration for 3D Point Clouds Yaru XIAN ${ }^{1}$, Jun XIAO ${ }^{1,}$, Ying $W_{A N G}{ }^{1}$, Mengyi SHAN, Chong ZHOU ${ }^{2}$ \\ ${ }^{1}$ University of the Chinese Academy of Sciences, No.19A Yuquan Road, Beijing, 100049, China \\ ${ }^{2}$ Information Center of China North Industries, Beijing, 100089, China \\ aemail: xiaojun@ucas.ac.cn
}

Keywords: Point Cloud; Scanner; Three-Dimensional Reconstruction; Registration

\begin{abstract}
D registration is an essential step in 3D reconstruction of real objects. In this paper, the fine registration methods for point clouds are classified systematically and analyzed theoretically. For several typical fine registration algorithms, experimental comparisons are also carried out on the effect of sampling parameters, iterations, iterative threshold and random noise. Furthermore, the stability and efficiency of the algorithms are tested. Based on the experimental results, the problems in current registration methods and the prospects of future research directions are also presented.
\end{abstract}

\section{Introduction}

$3 \mathrm{D}$ registration is a basic and crucial intermediate step of 3D data processing. It is a hot research area in the field of computer vision, 3D modeling, medical imaging, reverse engineering and remote sensing image processing etc. Registration is the precondition of reconstruction and many other applications, and its precision determines whether the follow-up work is successful or not.

According to whether an initial estimation is necessary, the current approaches can be divided into coarse registration and fine registration. Coarse registration is mainly used to calculate a rough estimate of the rigid transformation between two point clouds. The goal of fine registration is to get the transformation between two clouds as precise as possible. The method optimizes the transformation matrix by minimizing the predefined error function, resulting in high matching precision. Fine registration generally reduces the error function gradually by iteration. This paper mainly focuses on the fine registration methods.

The remainder is organized as follows. Firstly, fine registration techniques are classified and analyzed in Section 2. Next, results of comparative experiments of three typical registration algorithms are shown in Section 3. The paper ends with summaries and work prospects on the future.

\section{Registration Methods}

\section{Iterative Closest Points Algorithm}

The ICP method is by far the most popular registration method, which minimizes the predefined error function by an iterative optimization process, and each iteration of it can be divided into two steps, which are searching for the correspondence and calculating the transformation. ICP algorithm is of high accuracy and stability but low efficiency and requires an inclusion relation of the sets to ensure each point in the target set has a corresponding point in the reference set. This method searches for correspondence through geometric distances between pairs so that easy to fall into local optimum and sensitive to noise and outliers.

\section{Variants of the Iterative Closest Points Algorithm}

Many variants improve ICP to solve the drawbacks raised above and a detailed classification and comparison for some of them can be seen in [1], but it doesn't mention the multi-view registration and there are no real data used in the experiments. Next, we will introduce some variants of the ICP algorithm according to what they improve. 
- Sampling the Data

The ICP algorithm needs to find the corresponding point in the reference set for each point in the target set in each iteration, which is time consuming, so some algorithms sample the data to be registered to reduce the calculating time. There are some relatively simple sampling methods such as Uniform Sampling and Random Sampling, which may loss key features of the objects. The Normal-space sampling method is proposed and compared to the above sampling methods in [1], which tells us the that last one is superior to the other for flat surfaces, since this method reserves the detail feature of the data as far as possible based on the normal vector distribution of the points. The Covariance sampling method [2] is proposed based on the Normal-space sampling and is more stability and able to match symmetric surfaces effectively.

- Determining the Matches

There are many different kinds of correspondence searching methods in the variants of ICP:

1) Point-to-point. The ICP algorithm uses a point-to-point searching method, which is simple and intuitive, but is easy to produce large amount of error corresponds leading the registration into a local extreme. These methods often adopt some measures such as KD-tree to speed up the search.

2)Point-to-projection This method seeks for the projection on the reference set of target point from the viewpoint of the reference set to be the corresponding point of the target. This method is faster but less accurate than the prior one.

3)Point-to-surface This method is most widely used today, where the transformation is estimated by minimizing the sum of the squared distances from points to the corresponding surfaces. It is robust and of high precision. The most typical method in this type is the point-to-tangent plane method, which needs to calculate the projection of the point to the corresponding tangent plane, thus it is time-consuming. There are also other types of corresponding surface, such as NURBS [3] and B-spine [4] etc. These methods need fewer iterations and have high precision and efficiency. Literature [5] proposed a method of high efficiency and precision by combining the point-toprojection and point-to-tangent plane methods.

In addition, the local geometrical features such as the spherical harmonics [6] can also be used to search for the correspondence.

- Rejecting Point Pairs

There may be mismatched point pairs that need to be rejected in the pairs searched by the above methods. The easiest way is to define an absolute threshold or a relative percentage threshold and just reserve the pairs with small distances. The criteria used to reject mismatched pairs are generally the geometric properties and the invariance of the data, such as normal consistency and reciprocal correspondence. Literature [7] uses features of normal vector and curvature and so on, and literature [3] and picky ICP [8] use standard deviation.

- Calculating the transformation

In the ICP algorithm, the optimal coordinate transformation is usually calculated through a least squares method. The common methods to calculate the rotation matrix and the translation vector are the unit quaternion, singular value decomposition (SVD), orthogonal matrix method and the dual quaternion, the performances of these methods are similar [9].

- Judging convergence

An appropriate convergence criterion is crucial for the registration results. The criterion used in ICP is minimum mean square error (MMSE), and there are many different convergence criteria in the variants such as the distance of the two surfaces in the direction of the normal vector and main curvature [10]. Literature [11] constructs a quick and robust error function by combining the point-to-point and point-to-surface function. The Levenberg-Marquardt-Iterative Closest Point (LM-ICP) [12] algorithm optimizes the squared residual by applying the Levenberg-Marquardt (LM) method, thus to make the algorithm more robust.

Most of the variants of the ICP algorithm can only improve one of the steps in the whole process, and do not make up the flaws in ICP fundamentally, such as the local optimization etc. 


\section{The Method Based on Statistics}

The Normal Distribution Transform (NDT) [13] algorithm registers the clouds based on statistical information of the data instead of points. In this method, the probability distribution is calculated by the normal distribution of the points, and then the registration results are optimized via standard optimization techniques. The NDT algorithm is more efficient than ICP.

\section{The Method Based on Genetic Algorithm}

Genetic algorithms (GA) are search procedures learned from nature and bases on mechanics of natural selection and genetics. In [14], the authors present a dynamic genetic algorithm. Literature [15] proposes to use surface interpenetration measure (SIM) as the fitness function which is more robust based on genetic algorithms, and uses hill climbing algorithm to accelerate, which improves the result. Literature [16] summarizes and analyzed the similarities and differences among varieties of genetic algorithms, and compares them with the ICP algorithm. These methods are good at dealing with complex nonlinear problems and effectively avoid the local minimum and carry on multi view registration. However, generally these kinds of methods need lots of calculations and a long time to converge.

\section{The Method Based on Image}

In some applications, the registration of 3D point clouds and 2D images is often required, and there are many methods to meet the demand [17] [18]. Some panoramic scanning point cloud data can use panoramic image to register, and thereby combine the feature extraction of 2D image. In [19], the authors compare the generation, feature extraction and description of several kinds of panoramic image in detail. Paper [20] uses the correspondence of point cloud and image to process the point cloud registration by image registration. This kind of algorithm applies not only the coordinates of the point cloud but also the RGB color information or intensity information, thus to ensure the accuracy of the correspondence. However, the registration accuracy may not be high because of the resolution difference between three-dimensional point cloud data and optical image, camera calibration errors, as well as the angle deviation of scanner and camera.

Many earlier registration algorithms for range image can be seen in literature [21].

\section{Experimental Comparison and Analysis}

There is an experimental comparison of the ICP, LM-ICP and NDT algorithm in this section, and the data used in this paper is the Stanford Bunny provided by Stanford University Computer Graphics Laboratory, which was acquired by a Cyberware 3030 MS in 1994. All experiments are carried out by $\mathrm{C}++$ combined with PCL that runs on Microsoft Windows 10 System, and time given below is all counted on a PC with an Intel (R) Core (TM) i3-2100 3.10 GHz CPU and 6 GB RAM.

The comparative indicators used in the experiments are the following two:

1) The registration time The time premium displayed on the screen after registration.

2) The Euclidean fitness score The sum of squared distances of the clouds provided by PCL.

\section{Effects of Sampling}

The effects of sampling parameters to these algorithms are shown in Figure 1. The result shows that when the surfaces are flat, the impacts of sample to these algorithms are negligible, but for uneven surfaces, the NDT is sensitive to sample while the other two are not. With the increase of the number of points used in registration, the registration times are increased in all of the methods, especially the NDT algorithm. 

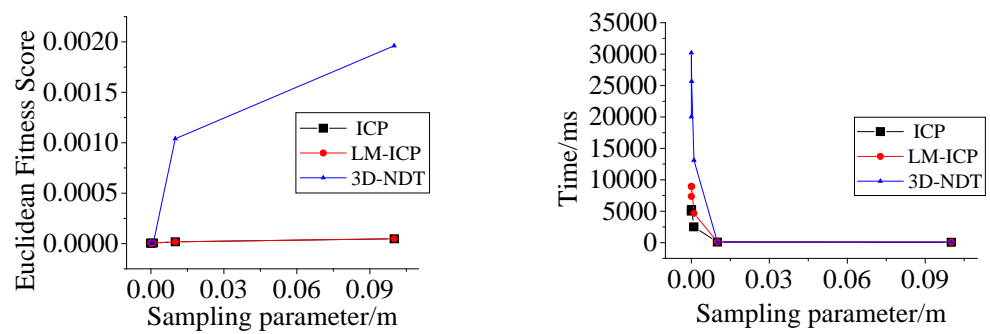

(a) The effects to fitness score (b) The effects to registration time

Fig.1. Effects of sampling parameters

\section{Effects of Iterations}

The effects of iterations to these algorithms are shown in Figure 2. A convergence threshold is set before registration, and the iteration stops if the threshold doesn't reach to the maximum number of iterations, otherwise it goes on until the maximum number. The result shows that ICP and LM-ICP tend to converge within ten iterations, so changes are small in the iterations out of ten. However, NDT converges until thirty iterations, so its convergence rate is slower than the other two.
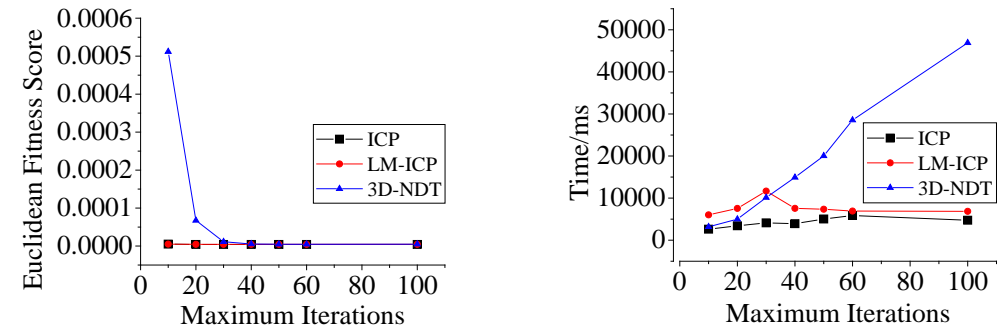

(a) The effects to fitness score (b) The effects to registration time Fig.2. Effects of iterations

\section{Effects of Terminating Threshold}

The effects of the threshold to these algorithms are shown in Figure 3. The experiments show that NDT can get a good result without a precise threshold though the other two need tighter criteria. However, a too tight criterion may cause registration to fail. The tighter criterion leads to longer times for these algorithms, but just a tighter result, that is, if the registration is unsuccessful, a tighter criterion just leads to a refined result in the false direction.
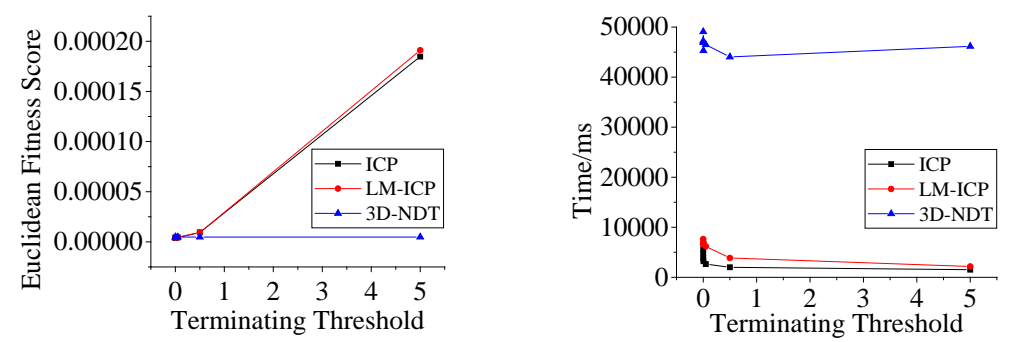

(a) The effects to fitness score (b) The effects to registration time

Fig.3. Effects of terminating threshold

\section{Effects of Noise}

The robustness of these algorithms is tested by adding random noise to source point cloud, and the experimental result is shown in Figure 4. We can see that the effect of noise is small if the amplitude is smaller than a certain value but significantly worse when the amplitude is greater than the value. The registration times of ICP and LM-ICP fluctuate within a range when noises are small, but tend to increase when noises are large. The registration time of NDT tends to decrease when the noise amplitude increases. 

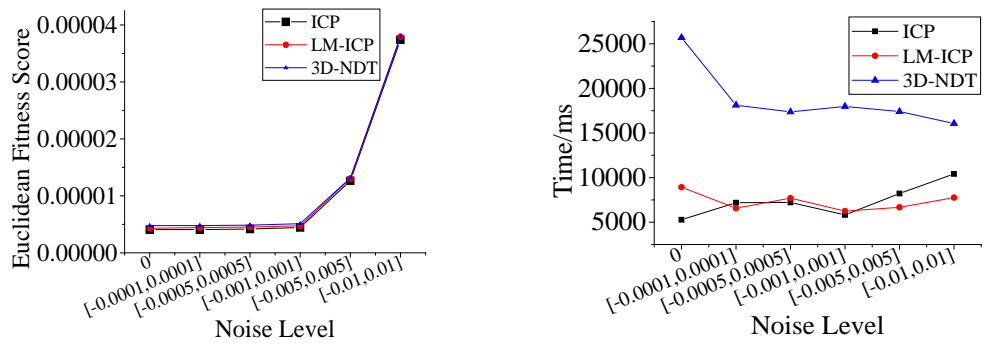

(a) The effects to fitness score (b) The effects to registration time

Fig.4. Effects of noise

The comparative experiments above show that the overall trend of fitness score and the time of ICP and LM-ICP are similar, though the registration time of LM-ICP is slightly longer than ICP. The NDT algorithm requires more iterations than the other two algorithms to converge when using the same parameters, but it has a lower requirement for the terminating threshold so we can set a larger threshold to accelerate. In addition, when the surfaces are flat, the NDT algorithm can achieve similar results as the other two algorithms with a significantly shorter time through voxel filter.

\section{Conclusion}

In this paper, the methods of fine registration are introduced in classification, and the advantages and disadvantages of each method are analyzed. From the research above, some issues need further study and discussion. Through a survey of the research status at home and abroad: (1) Most registration algorithms such as ICP and its various variants require good initial values otherwise they are easy to fall into local optimum; (2) Many algorithms require complex selection of parameters; (3) Most registration algorithms have bad results for partially overlapping point clouds, and the robustness to noise and outliers is worse; (4) Algorithms of multi-view registration are few, and most of the existing algorithms are more complex. Moreover, many current multi-view registration methods are based on a number of continuous pair-wise registrations, which will result in the accumulation of registration errors; (5) Current algorithms are mostly based on rigid transformation, but few use non-rigid transformation.

In summary, there is a big search space for improvement in registration. It is good to form an effective system of evaluation of the registration results, thus to compare the stand or fall of most registration algorithms. Then an open registration application platform can be constructed so that users can build an appropriate set of complete registration process by selecting the method used in every step according to their requirement. In addition, the current and subsequent algorithms should make the parameter selection as intelligent as possible, thus to improve the automation of registration.

\section{Acknowledgement}

This work is supported by the National Natural Science Foundation of China (No. 61471338), President Fund of UCAS, and Youth Innovation Promotion Association CAS (2015361).

\section{References}

[1] Rusinkiewicz S, Levoy M. Efficient variants of the ICP algorithm[C]. Proceedings of the Third IEEE International Conference on 3D Digital Imaging and Modeling, 2001: 145-152.

[2] Gelfand N, Ikemoto L, Rusinkiewicz S, et al. Geometrically stable sampling for the ICP algorithm[C]. Proceedings of the Fourth IEEE International Conference on 3-D Digital Imaging and Modeling, 2003: 260-267.

[3] Ristic M, Brujic D. Efficient registration of NURBS geometry[J]. Image and Vision Computing, 1997, 15(12): 925-935. 
[4] Tsai T H, Fan K C. An image matching algorithm for variable mesh surfaces[J]. Measurement, 2007, 40(3): 329-337.

[5] Park S Y, Subbarao M. A fast point-to-tangent plane technique for multi-view registration[C]. Proceedings of the Fourth IEEE International Conference on 3-D Digital Imaging and Modeling (3DIM), 2003: 276-283.

[6] Sharp G C, Lee S W, Wehe D K. ICP registration using invariant features[J]. IEEE Transactions on Pattern Analysis and Machine Intelligence, 2002, 24(1): 90-102.

[7] Zhu Y J, Zhou L Y, Zhang L Y. Registration of Scattered Cloud Data[J]. Journal of Computer-Aided Design \& Computer Graphics, 2006, 18(4): 475-481.

[8] Zinßer T, Schmidt J, Niemann H. A refined ICP algorithm for robust 3-D correspondence estimation[C]. Proceedings of IEEE International Conference on Image Processing (ICIP), 2003, 2: II-695- II-698.

[9] Eggert D W, Lorusso A, Fisher R B. Estimating 3-D rigid body transformations: a comparison of four major algorithms[J]. Machine Vision and Applications, 1997, 9(5-6): 272-290.

[10] Kase K, Makinouchi A, Nakagawa T, et al. Shape error evaluation method of free-form surfaces[J]. Computer-Aided Design, 1999, 31(8): 495-505.

[11] Mitra N. Algorithms for comparing and analyzing 3D geometry[D]. Stanford University, 2006.

[12] Fitzgibbon A W. Robust registration of 2D and 3D point sets[J]. Image and Vision Computing, 2003, 21(13): 1145-1153.

[13] Magnusson M, Lilienthal A, Duckett T. Scan registration for autonomous mining vehicles using 3D-NDT[J]. Journal of Field Robotics, 2007, 24(10): 803-827.

[14] Chow C K, Tsui H T, Lee T. Surface registration using a dynamic genetic algorithm[J]. Pattern recognition, 2004, 37(1): 105-117.

[15] Silva L, Bellon O R P, Boyer K L. Enhanced, robust genetic algorithms for multiview range image registration[C]. Proceedings of the Fourth IEEE International Conference on 3-D Digital Imaging and Modeling (3DIM), 2003: 268-275.

[16] Santamaría J, Cordón O, Damas S. A comparative study of state-of-the-art evolutionary image registration methods for 3D modeling[J]. Computer Vision \& Image Understanding, 2011, 115(9):1340-1354.

[17] $\mathrm{Hu} \mathrm{X,} \mathrm{Ye} \mathrm{L,} \mathrm{Li} \mathrm{X,} \mathrm{et} \mathrm{al.} \mathrm{Fusion} \mathrm{of} \mathrm{airborne} \mathrm{lidar} \mathrm{point} \mathrm{cloud} \mathrm{and} \mathrm{imagery} \mathrm{captured} \mathrm{from}$ integrated sensor system[C]. International Symposium on Lidar and Radar Mapping Technologies: International Society for Optics and Photonics, 2011: 209-224.

[18] Crombez N, Caron G, Mouaddib E. 3D POINT CLOUD MODEL COLORIZATION BY DENSE REGISTRATION OF DIGITAL IMAGES[J]. The International Archives of Photogrammetry, Remote Sensing and Spatial Information Sciences, 2015, 40(5): 123-130.

[19] Houshiar H, Elseberg J, Borrmann D, et al. A study of projections for key point based registration of panoramic terrestrial 3D laser scan[J]. Geo-spatial Information Science, 2015, 18(1): 11-31.

[20] Yang M Y, Cao Y, McDonald J. Fusion of camera images and laser scans for wide baseline 3D scene alignment in urban environments[J]. International Society for Photogrammetry and Remote Sensing (ISPRS) Journal of Photogrammetry and Remote Sensing, 2011, 66(6): S52-S61.

[21] Salvi J, Matabosch C, Fofi D, et al. A review of recent range image registration methods with accuracy evaluation[J]. Image and Vision computing, 2007, 25(5): 578-596. 